Cahiers québécois de démographie

\title{
La mortalité différentielle mesurée en termes de dissemblance
} Les écarts de mortalité entre sexes selon les tables italiennes du $\mathrm{XX}^{\mathrm{e}}$ siècle

\section{Differentials in Mortality Measured in Terms of Dissimilarity} Mortality Differences between Sexes According to the Italian Tables of the 20th Century

\section{La mortalidad diferencial medida en términos de desemejanza.} Las diferencias de mortalidad entre los sexos segun las tablas italianas del siglo XX

\section{Carlo Maccheroni}

Volume 17, numéro 1, printemps 1988

Politiques de population

URI : https://id.erudit.org/iderudit/600630ar

DOI : https://doi.org/10.7202/600630ar

Aller au sommaire du numéro

Éditeur(s)

Association des démographes du Québec

ISSN

0380-1721 (imprimé)

1705-1495 (numérique)

Découvrir la revue

Citer cet article

Maccheroni, C. (1988). La mortalité différentielle mesurée en termes de dissemblance : les écarts de mortalité entre sexes selon les tables italiennes du $\mathrm{XX}^{\mathrm{e}}$ siècle. Cahiers québécois de démographie, 17(1), 65-89.

https://doi.org/10.7202/600630ar
Résumé de l'article

La comparaison des espérances de vie peut, sous certaines conditions, conduire à une mesure bien plus précise, à savoir celle de la dissemblance entre les distributions empiriques des durées de survie mises en confrontation. Cet article examine les conditions qui doivent être réunies pour qu'on puisse accepter cette interprétation de la comparaison des espérances de vie, et applique cette approche aux données des tables de mortalité italiennes du $\mathrm{XX}^{\mathrm{e}}$ siècle.
Tous droits réservés @ Association des démographes du Québec, 1988

Ce document est protégé par la loi sur le droit d'auteur. L'utilisation des services d'Érudit (y compris la reproduction) est assujettie à sa politique d'utilisation que vous pouvez consulter en ligne.

https://apropos.erudit.org/fr/usagers/politique-dutilisation/ 
Cahiers québécois de démographie

Vo1. 17, no 1, printemps 1988

\title{
La mortalité différentielle mesurée en termes de dissemblance. Les écarts de mortalité entre sexes selon les tables italiennes du XXe siècle
}

\author{
Carlo MACCHERONI*
}

\section{INTRODUCTION}

Les mesures les plus fréquemment utilisées dans les études de la mortalité sont les taux spécifiques de mortalité, les probabilités de décès, l'espérance de vie ou vie moyenne. De 1'efficacité avec laquelle ces indicateurs expriment l'intensité du risque de décès, et de l'analyse comparée des résultats obtenus avec ces indicateurs, dépendra la capacité de la démographie à identifier et prévoir les soi-disants groupes à risques, à évaluer l'impact des mesures politiques, et à développer des pistes de recherche interdisciplinaire.

Les taux et probabilités d'une part et 1'espérance de vie d'autre part, répondent à des problématiques différentes, les premières étant des mesures spécifiques (par âge), la seconde représentant au contraire une mesure synthétique résumant l'expérience de mortalité sur un segment plus ou moins long de la vie. La référence à la vie moyenne facilite sans doute l'interprétation et la comparaison d'expériences diverses, mais implique en général, outre le renoncement à connaître la pluralité des résultats empiriques qui la sous-tendent, la mise en lumière d'une seule caractéristique statistique, à savoir la soi-disante "tendance centrale" de chaque ensemble de grandeurs considérées. Il importe toutefois de souligner que l'analyse menée en termes d'espérance de vie ne se réduit pas nécessairement à une simple comparaison "en moyenne", mais peut, sous certaines conditions, conduire à une mesure bien plus précise, à savoir celle de la dissemblance entre les distributions empiriques des durées de survie qui, plus ou moins explicitement, sont mises en confrontation.

* Université Bocconi, Institut de méthodes quantitatives, Milan. 
La première section de cet article est consacrée à l'examen des conditions qui doivent être réunies pour qu'on puisse accepter cette interprétation de la comparaison entre espérances de vie. Sur la base de ces précisions, nous pourrons alors, dans les deux sections suivantes, procéder à une analyse de 1a mortalité différentielle en termes de dissemblance. Nous avons choisi d'examiner la mortalité différentielle selon le sexe, plutôt que d'autres types d'analyse différentielle, parce qu'il s'agit là d'un domaine de recherche qui a joué et joue un rôle important en démographie. En outre, nous avons choisi d'appliquer notre approche aux données italiennes parce que pour ce pays, dès les années 1950, la mesure de la dissemblance entre les variables qui décrivent la durée de survie de deux sexes colncide toujours avec la différence absolue des espérances de vie correspondantes, et par conséquent, elle est directement dérivable des tables de mortalité disponibles depuis cette période. Il est cependant apparu intéressant de déterminer le niveau de dissemblance également à partir des tables des périodes antérieures, en remontant jusqu'au début du siècle, afin de pouvoir étudier comment ont évolué dans le long terme, et d'un point de vue qui nous semble nouveau, les durées de survie des deux sexes.

1. LA DISSEMBLANCE ENTRE LES DISTRIBUTIONS RELATIVES AUX DURÉES DE SURVIE

Le problème de la comparaison entre distributions empiriques avait déjà été traité par Gini (1914), à qui nous devons précisément les fondements de l'analyse de dissemblance. L'approche que nous proposons ici pour vérifier sous quelles conditions la comparaison entre espérances de vie correspond à la dissemblance entre les variables dont l'espérance de vie est la valeur attendue, est directement liée à la tradition de 1 'analyse démographique. En effet, nous utilisons des fonctions biométriques connues et aisément dérivables de la table de mortalité.

Soit $\mathrm{Z}$ la variable générique "nombre d'années qui restent à vivre à compter du $x$-ième anniversaire" :

$\mathrm{z}_{\mathrm{x}} \equiv\left\{\begin{array}{cccc}0-1 & 1-2 & \cdots & {[(\omega-1)-\mathrm{x}]-(\omega-\mathrm{x})} \\ \frac{\mathrm{d} \mathrm{x}}{1 \mathrm{x}} & \frac{\mathrm{d}(\mathrm{x}+1)}{1 \mathrm{x}} & \cdots & \frac{\mathrm{d}(\omega-1)}{1 \mathrm{x}}\end{array}\right.$ 
où $\mathrm{x}=0,1, \ldots,(\omega-1)$, avec $(\omega-1)$ représentant 1'âge final, celui pour lequel $1 \omega=0$,

$\mathrm{dx}=1 \mathrm{e}$ nombre de décès à $I^{\prime}$ âge $\mathrm{x}$,

$1 \mathrm{x}=1 \mathrm{e}$ nombre de survivants d'âge $\mathrm{x}$,

et dont la valeur attendue est $\varepsilon_{x}$, l'espérance de vie.

Désignons par A et $B$ deux sous-ensembles disjoints de la population, ou deux populations différentes, pour lesquels il est possible de déterminer respectivement $\mathrm{Z}_{\mathrm{x}}^{(\mathrm{A})}$ et $\mathrm{Z}_{\mathrm{x}}^{(\mathrm{B})}$, et par $\Delta$ $1^{\prime}$ indice de dissemblance entre les variables $z_{x}^{(A)}$ et $z_{x}^{(B)}$. D'une
façon générale, on a

$$
\Delta=D\left(z_{x}^{(A)}, z_{x}^{(B)}\right) \geqq\left|\stackrel{o}{e}_{x}^{(A)}-\frac{o}{e_{x}^{(B)}}\right|
$$

A cette dernière relation sont liés deux résultats connus, que nous allons reformuler en termes démographiques.

Le premier résultat est que la dissemblance entre deux distributions est représentée par l'étendue de la surface comprise entre leurs fonctions de répartition (Cifarelli et Moeller, 1981; Leti, 1983). Si, par exemple, $\mathrm{X}^{(\mathrm{A})}$ et $\mathrm{X}^{(\mathrm{B})}$ sont les deux variables considérées, et si nous représentons graphiquement leurs fonctions de répartition $F(x)$ et $G(x)$ comme étant

$$
F(x)=\operatorname{Pr}\left\{x^{(A)}<x\right\} \text { et } G(x)=\operatorname{Pr}\left\{x^{(B)}<x\right\} \text {, }
$$

alors 1a mesure de dissemblance $\Delta=D\left(X^{(A)}, X^{(B)}\right)$ correspond, en termes géométriques, à la partie hachurée de la figure 1 , avec

$$
\Delta=D\left(X^{(A)}, X^{(b)}\right)=a+b+c
$$

Le second résultat auquel nous nous référons concerne une représentation peu courante de la moyenne arithmétique ou valeur attendue, représentation qui est cependant particulièrement pertinente en ce qui nous concerne, car elle permet d'exprimer la moyenne arithmétique en termes de fonction de répartition. Plus précisément, si $X$ est une variable dont m est $1 \mathrm{a}$ valeur attendue, on peut démontrer (Mood et alii, 1974) que

$$
\mathrm{m}=\mathrm{E}\{\mathrm{X}\}=\int_{0}^{\infty}[1-\mathrm{F}(\mathrm{x})] \mathrm{dx}-\underline{-}_{-\infty} \mathrm{F}(\mathrm{x}) \mathrm{dx}
$$


Figure 1

Représentation graphique de la dissemblance entre deux distributions

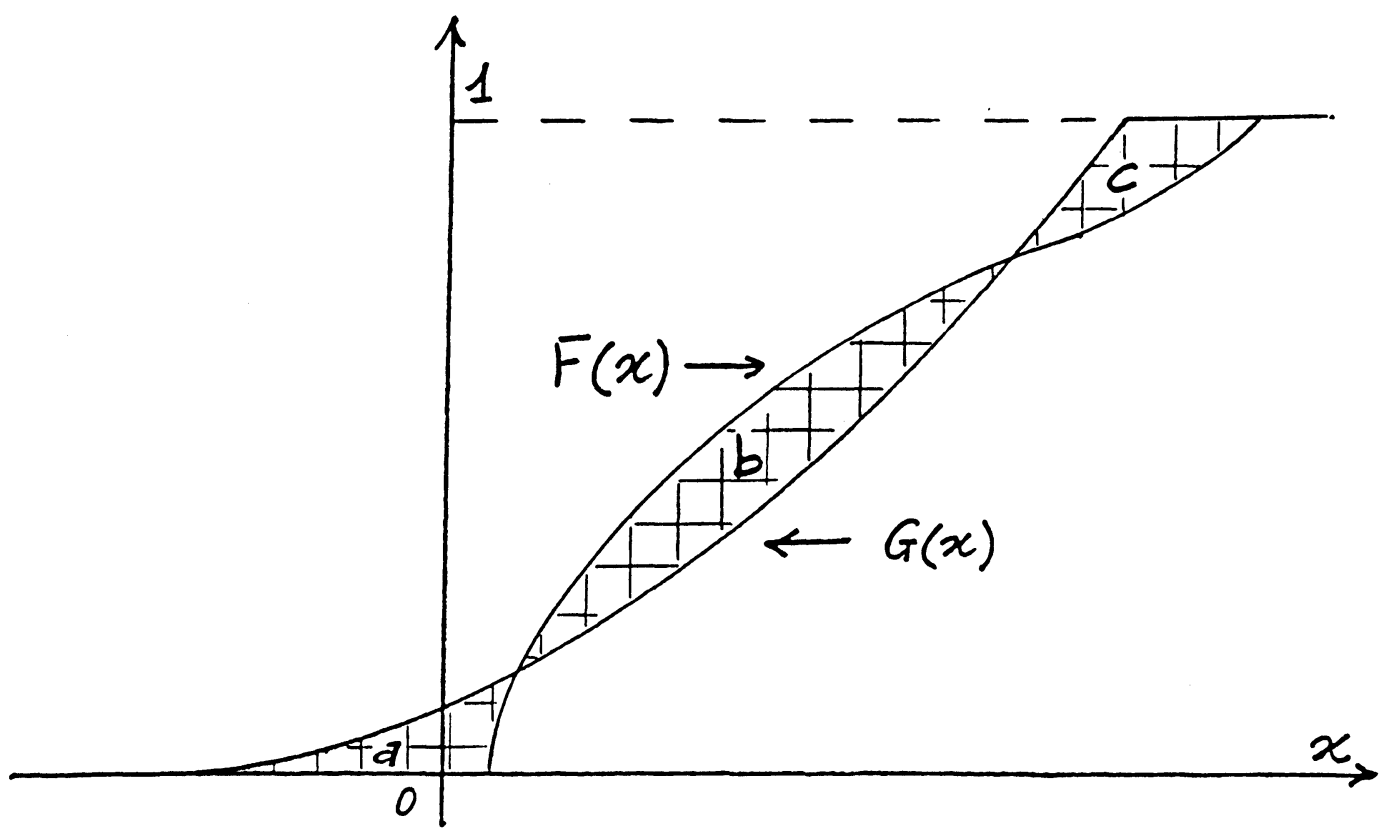

Figure 2

La moyenne arithmétique en termes de fonction de répartition

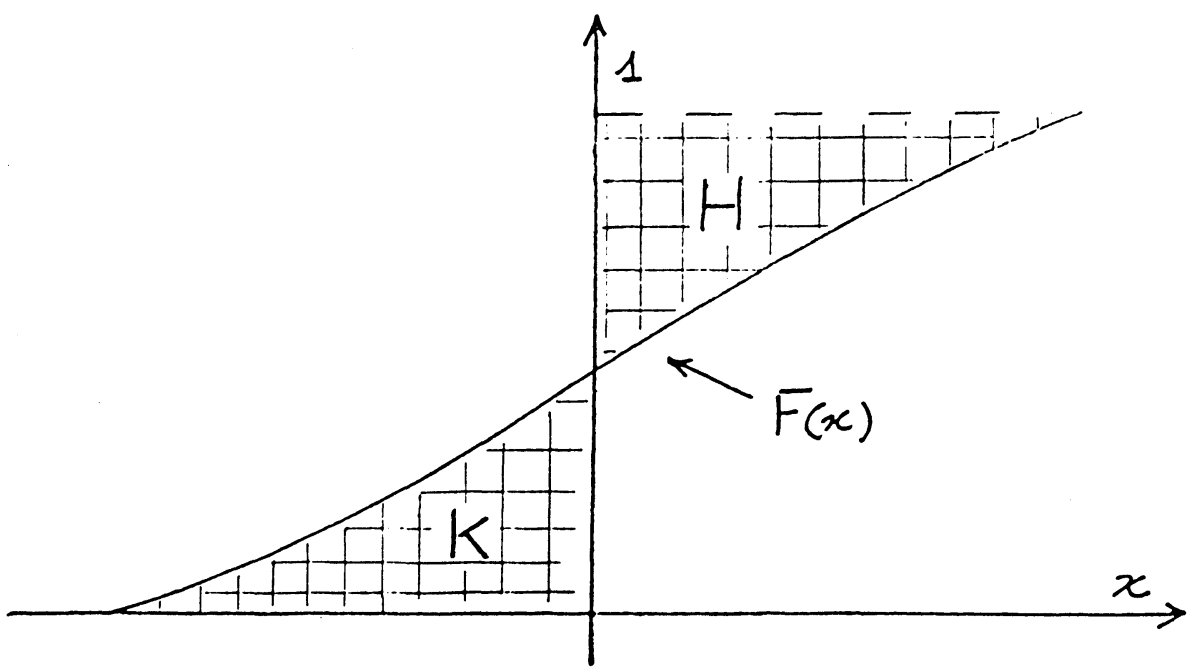


où $F(x)$ est la fonction de répartition de $X$. La moyenne correspond donc à la différence entre les deux aires $H$ et $K$, telles qu'illustrées par la figure 2.

Si nous reprenons ces deux résultats en les interprétant en termes de notre variable "nombre d'années qui restent à vivre" (équation 1 ), et si l'on tient compte de ce que $Z$ est de toute évidence une variable positive et que sa fonction de répartition

$$
F_{Z_{x}}(n)={ }_{n}{ }^{x} \quad(n=1,2, \ldots, \omega-x)
$$

représente la probabilité, pour un individu d'âge $x$, d'avoir une durée de survie inférieure à $n$ années, ou encore la probabilité de mourir avant d'avoir $(x+n)$ ans révolus, alors on peut affirmer que

$1^{\circ} 1^{\prime}$ espérance de vie $\&$, déterminée sur la base de la relation (3), coincide avec ${ }^{\prime}$ l'étendue de la surface hachurée de la figure 3 .

Figure 3

Espérance de vie et probabilité de mourir

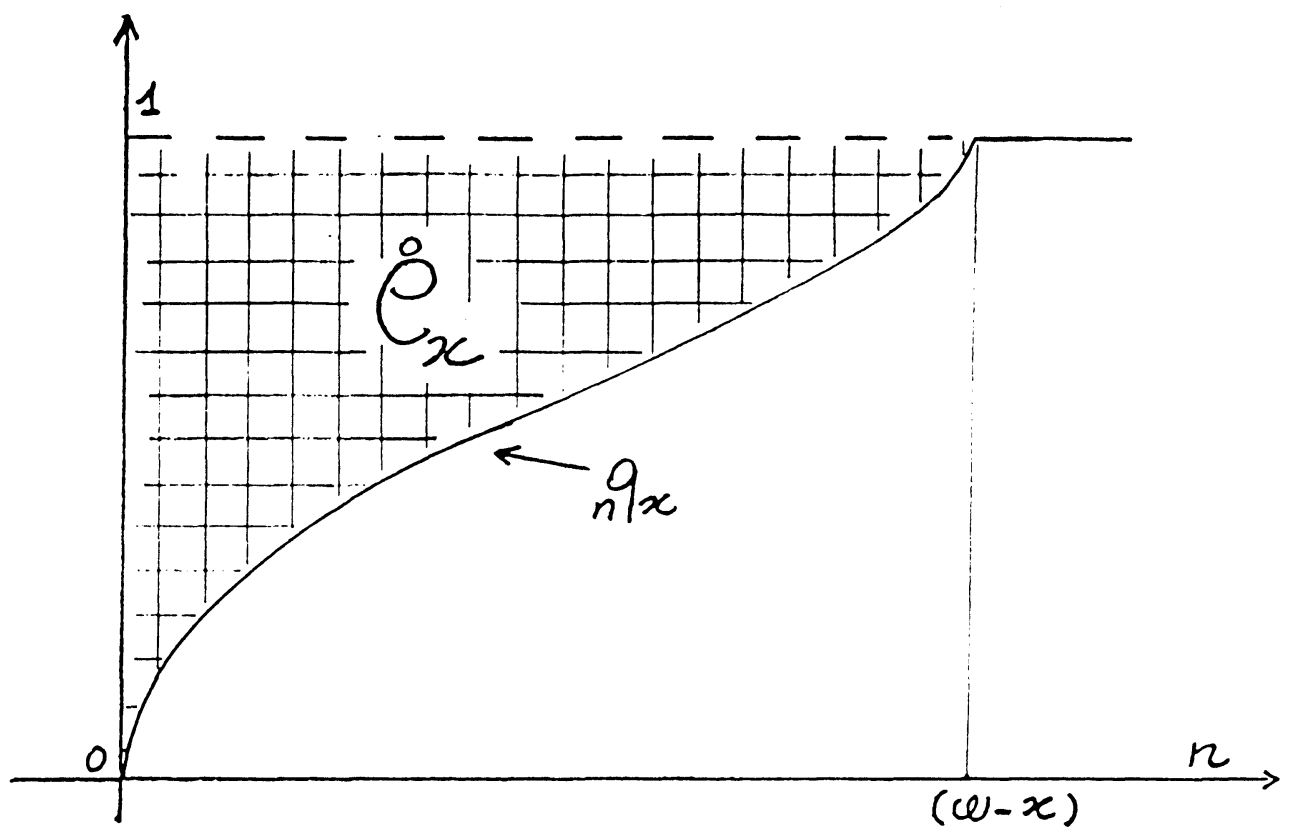


$2^{\circ}$ La comparaison entre les distributions $\mathrm{Z}_{\mathrm{x}}^{(\mathrm{A})}$ et $\mathrm{z}_{\mathrm{x}}^{(\mathrm{B})}$, et donc la relation entre les indices de dissemblance, d'une part, et la différence absolue entre les moyennes, d'autre part, donne lieu aux trois cas illustrés par la figure 4.

Plus précisément :

a) Lorsque les fonctions de répartition $n^{q_{x}^{(A)}}$ et $n_{x}^{(B)}$ de $z_{x}^{(A)}$ et $\mathrm{Z}_{\mathrm{X}}^{(\mathrm{B})}$ se coupent, comme dans le cas illustré par la figure 4.a, on $a$

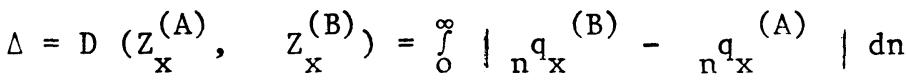

$$
\begin{aligned}
& =\int_{0}^{\infty}\left|\left(1-n^{q} x^{(A)}\right)-\left(1-n^{q} x^{(B)}\right)\right| d n \\
& \geqq\left|\int_{0}^{\infty}\left(1-n^{q} x^{(A)}\right) d n-\int_{0}^{\infty}\left(1-n^{q} x^{(B)}\right) d n\right|
\end{aligned}
$$

Or, même si la limite supérieure de toute intégrale doit être $(\omega-x)$ (puisque l'âge extrême est fini), on peut également intégrer entre zéro et l'infini, parce que pour $\mathrm{n}>(\omega-\mathrm{x})$ l'intégrant est nul. L'équation (4) est donc égale à la suivante :

$$
\begin{aligned}
& \Delta=\int_{0}^{\infty}\left|{ }_{n} p_{x}^{(A)}-{ }_{n} p_{x}^{(B)}\right| d n \geqq\left|\int_{0}^{\infty} n_{n} p_{x}^{(A)} d n-\int_{0}^{\infty}{ }_{n} p_{x}^{(B)} d n\right|= \\
& =\mid \stackrel{\circ}{e}_{x}^{(A)}-\stackrel{o}{e}_{x}^{(B)}
\end{aligned}
$$

b) Lorsque les distributions $\mathrm{Z}_{x}^{(A)}$ et $\mathrm{z}_{\mathrm{x}}^{(B)}$ sont semblables, on a $n^{q}{ }^{(A)}=n^{q} x^{(B)}$ pour tout $n$, et donc évidemment

$\stackrel{o}{x}_{x}^{(A)}=\stackrel{\circ}{x}_{(B)}^{(B)}$ avec $\Delta=0$ (figure 4.b) 
Graphique 4

Les trois cas de dissemblance entre les distributions du "nombre d'années qui restent à vivre à compter du $x$-ième anniversaire"

a)

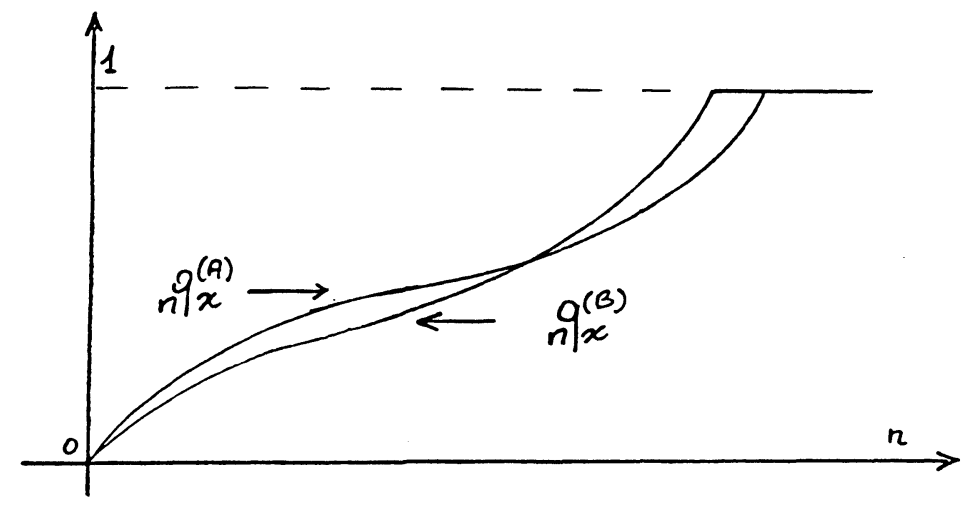

b)

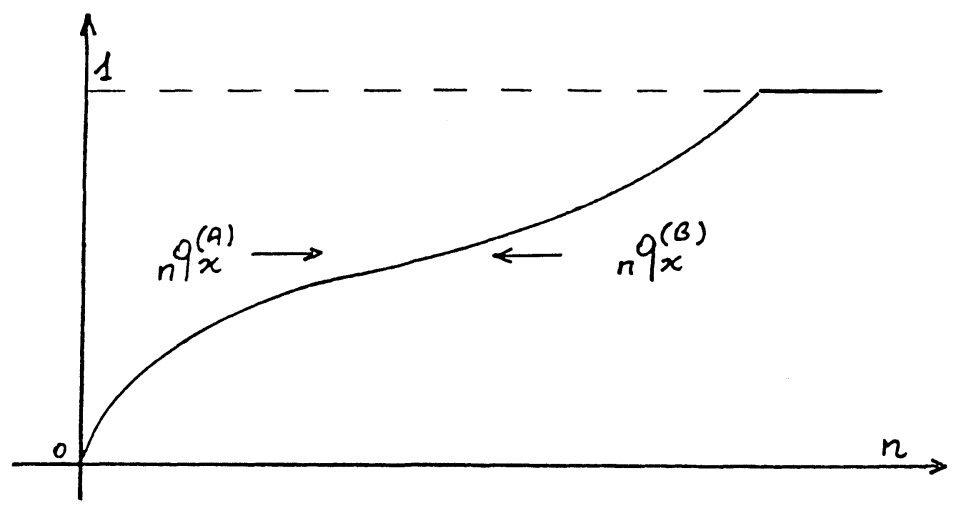

c)

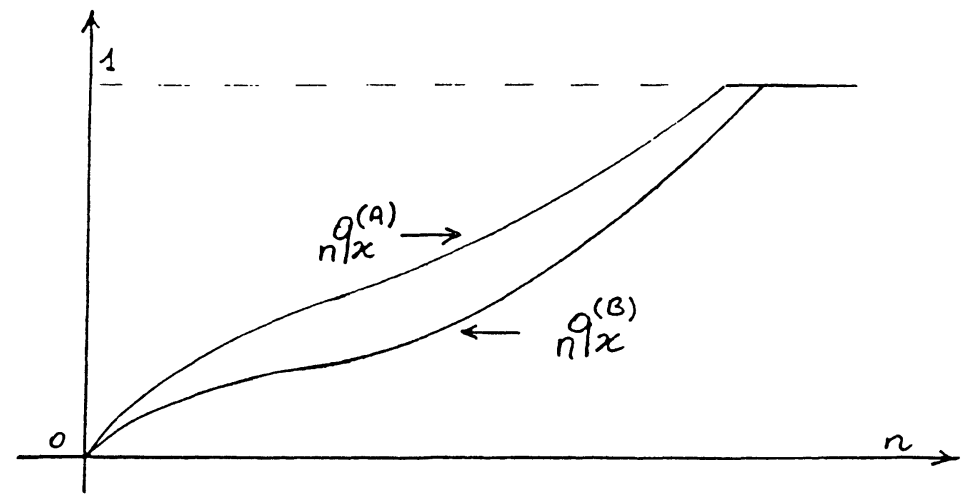


C) Lorsque les fonctions de répartition ne se coupent pas, comme dans le cas illustré par la figure 4.c, où ${ }_{n} q^{(B)}<n^{q}{ }^{(A)}$ pour tout $n$, alors $Z_{x}(B)$ est stochastiquement ou statistiquement plus grand que $z_{x}^{(A)}$, et donc $\stackrel{o}{x}_{x}^{(B)}>e_{x}^{(A)}$. L'équation (5) devient alors

$$
\begin{aligned}
\Delta=\int_{0}^{\infty}\left|{ }_{n} p_{x}^{(A)}-{ }_{n} p_{x}^{(B)}\right| \mathrm{dn} & =\left|\int_{0}^{\infty}{ }_{n} p_{x}^{(A)} \mathrm{dn}-\int_{0}^{\infty}{ }_{n} p_{x}^{(B)} \mathrm{dn}\right| \\
& =\left|\stackrel{o}{e_{x}^{(A)}}-\underset{x}{\stackrel{o}{(B)}}\right|
\end{aligned}
$$

Dans ce cas, la connaissance de $\Delta$ ne sert évidemment pas à intégrer celle obtenue de la comparaison entre les deux espérances de vie.

2. LA MORTALITE DIFFÉRENTIELLE PAR SEXE EN TERMES DE DISSEMBLANCE

Les caractéristiques de la différenciation progressive de la durée de vie des deux sexes ont été analysées en utilisant les tables de mortalité italiennes construites depuis le début de ce siècle jusqu'à celle de 1930-1932, et, pour la période plus récente, à partir des tables de 1950-1953, 1960-1962, 1970-1972 et 1977-1979.

De chacune de ces tables ont été dérivées les mesures "abrégées" par groupe quinquennal d'âge, et à partir de ces dernières ont été construites les fonctions de répartition ${ }_{n}{ }^{(H)}$ et ${ }_{n} q_{x}^{(F)} \quad(n=5,10, \ldots, \omega-x)$ pour les variables $z_{x}^{(H)}$ et $z_{x}^{(F)}(x=0,5, \ldots, 65)$, relatives respectivement aux hommes (H) $x$ et aux femmes (F). Dans la détermination de la dissemblance entre les deux distributions, nous avons distingué tantôt l'âge 1, tantôt l'âge 0 , à cause de 1 a bien connue non-homogénéité des conditions de mortalité à l'intérieur du premier groupe quinquennal d'âge. De la comparaison entre ${ }_{n} q_{x}^{(H)}$ et $n^{q}{ }_{x}^{(F)}$, on passe ensuite à celle entre les deux relations utilisées pour mesurer la dissemblance, soit celle de 1 'équation (5), soit celle de 1 'équation (6). 
I1 est bien connu que la surmortalité masculine, en tant que phénomène commun à tous les âges, s'est manifestée en Italie à partir des tables de 1951-1953. Par contre, dans les tables antérieures, Ia comparaison entre les $q_{x}^{(H)}$ et les $q_{x}^{(F)}$ révélait une surmortalité féminine à 1 'adolescence, à quelques âges juvéniles et dans les premiers âges adultes (25-35 ans), avec une surmortalité masculine aux autres âges (voir Di Comite, 1974, et Natale, 1979). Nos fonctions de répartition ${ }_{\mathrm{n}} \mathrm{q}_{\mathrm{x}}^{(\mathrm{H})}$ et $\mathrm{n}_{\mathrm{x}}^{(\mathrm{F})}$ reflètent évidemment ces mêmes différences, car les fonctions se coupent (figure 4.a) seulement dans les cas où et pour les groupes d'âge où s'est manifestée une surmortalité féminine. Il s'ensuit que pour le premier groupe de tables de mortalité (celles antérieures à la dernière guerre mondiale), la dissemblance a été déterminée, selon les cas, sur la base de la relation (5) ou de la relation (6), alors que pour le second groupe de tables (celles de l'après-guerre), seule la relation (6) a été utilisée.

Le fait d'avoir pris en considération les fonctions de répartition de chaque variable $\mathrm{z}_{\mathrm{x}}^{(\mathrm{H})}$ et $\mathrm{z}_{\mathrm{x}}^{(\mathrm{F})} \quad(\mathrm{x}=0,5 \ldots, 65)$ également pour les tables de $1^{\prime}$ après-guerre résulte de notre souci de joindre à 1 'analyse des différences entre sexes pour un âge donné une analyse correspondante par âge pour chaque sexe. Ceci nous a permis de constater que, pour chaque sexe et pour toutes les tables utilisées, on a toujours $\mathrm{Z}_{\mathrm{x}}>\mathrm{Z}_{\mathrm{x}+5}$ seulement à partir des âges centraux (25-35 ans), alors que pour les âges antérieurs, I'inégalité $Z_{x}>Z_{x+5}$ ne vaut que pour quelques âges de l'adolescence et sans qu'il n'y ait quelque uniformité entre les sexes. Il s'ensuit que la comparaison entre les espérances de vie à deux âges successifs ne reflètent pas, en général, la distance qui sépare les ensembles représentatifs des durées de survie à ces âges. Ces précisions sur la forme des fonctions de répartition nous permettent de nous limiter dorénavant à $l^{\prime}$ examen des résultats obtenus de la seule analyse de dissemblance.

Le premier groupe de résultats que nous analyserons permet de voir comment ont divergé progressivement les distributions des durées de survie des deux sexes, processus qui s'est manifesté avec une intensité particulière au cours des périodes où la mortalité baissait le plus. Le tableau 1 et la figure 5 montrent que cette augmentation, à chaque âge, de la distance entre les ensembles représentatifs de la durée de survie des hommes et des femmes, s'est manifestée en trois phases. 
Dissemblance (en années) entre les variables "nombre d'années qui restent à vivre à compter du x-ième anniversaire" relatives à chaque sexe, selon 1'âge. Italie, 1899-1902 à 1977-1979

\begin{tabular}{rcccccccc}
\hline Age & $1899-1902$ & $1910-1912$ & $1921-1922$ & $1930-1932$ & $1950-1953$ & $1960-1962$ & $1970-1972$ & $1977-1979$ \\
\hline 0 & 0,57 & 0,76 & 1,48 & 2,24 & 3,53 & 5,03 & 5,91 & 6,58 \\
1 & 0,80 & 0,71 & 0,77 & 1,61 & 3,12 & 4,66 & 5,62 & 6,39 \\
5 & 0,77 & 0,77 & 0,74 & 1,82 & 3,14 & 4,66 & 5,61 & 6,36 \\
10 & 0,71 & 0,86 & 0,75 & 1,83 & 3,11 & 4,61 & 5,57 & 6,33 \\
15 & 0,65 & 0,72 & 0,77 & 1,84 & 3,05 & 4,57 & 5,50 & 6,29 \\
20 & 0,58 & 0,70 & 0,83 & 1,88 & 2,97 & 4,40 & 5,34 & 6,14 \\
25 & 0,59 & 0,73 & 0,77 & 1,92 & 2,88 & 4,23 & 5,19 & 5,98 \\
30 & 0,56 & 0,74 & 0,81 & 1,83 & 2,81 & 4,11 & 5,08 & 5,87 \\
35 & 0,63 & 0,82 & 0,85 & 1,80 & 2,75 & 4,01 & 4,99 & 5,78 \\
40 & 0,74 & 0,88 & 0,88 & 1,75 & 2,70 & 3,91 & 4,87 & 5,67 \\
45 & 0,76 & 0,85 & 0,86 & 1,63 & 2,55 & 3,76 & 4,67 & 5,49 \\
50 & 0,59 & 0,68 & 0,72 & 1,44 & 2,30 & 3,53 & 4,40 & 5,19 \\
55 & 0,36 & 0,50 & 0,59 & 1,21 & 1,93 & 3,16 & 3,82 & 4,72 \\
60 & 0,14 & 0,26 & 0,35 & 0,97 & 1,49 & 2,62 & 3,50 & 4,14 \\
65 & 0,07 & 0,11 & 0,19 & 0,74 & 1,08 & 1,95 & 2,85 & 3,40 \\
\hline
\end{tabular}


Figure 5

Dissemblance (en années) entre les variables "nombre d'années qui restent à vivre à compter du x-ième anniversaire" relatives à chaque sexe, selon l'âge. Italie, 1899-1902 à 1977-1979

$$
D\left(z_{x}^{m}, t_{x}^{n}\right)
$$

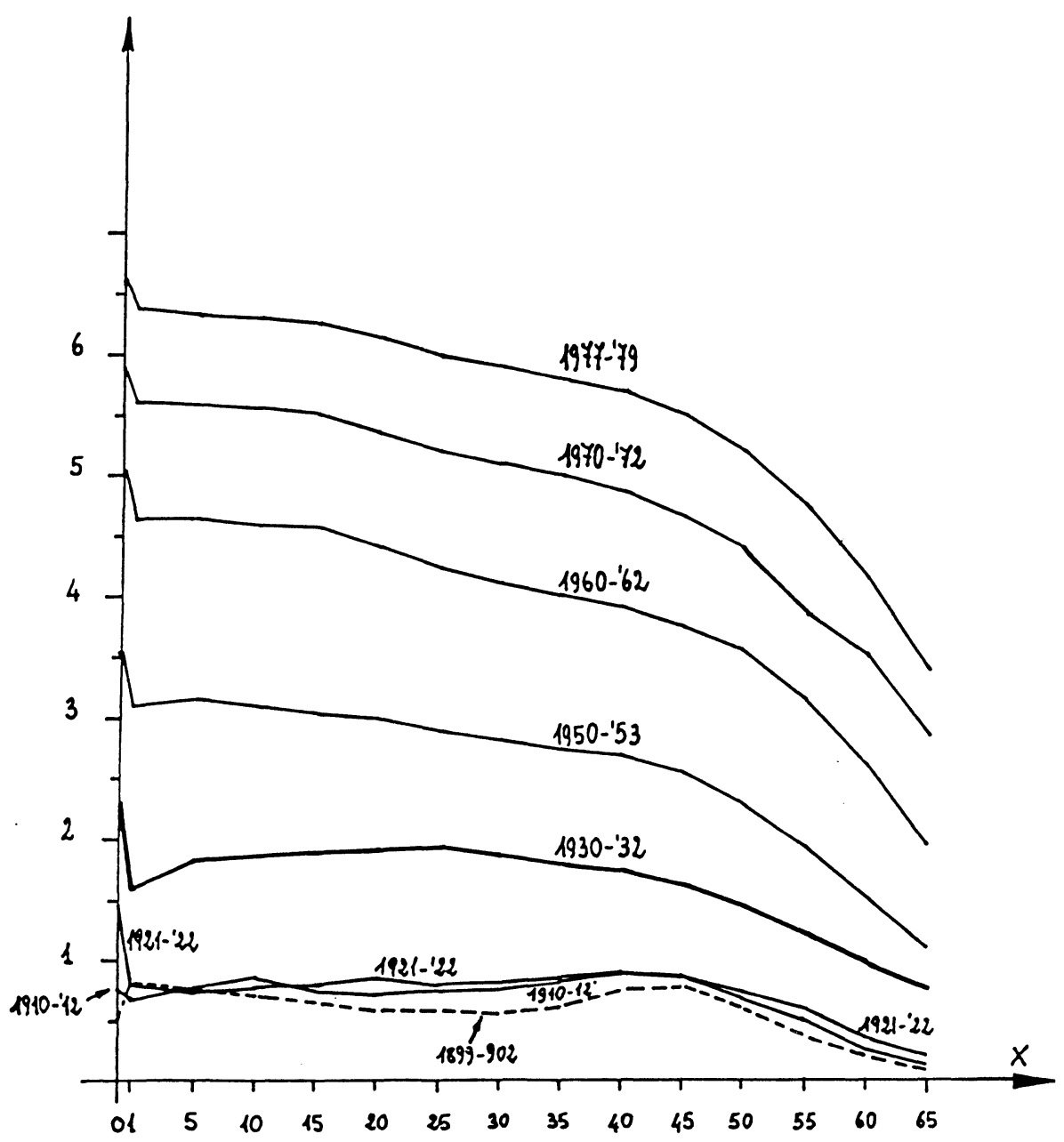


La première phase se termine avec la table de 1910-1912, et est caractérisée par une faible divergence entre les durées de survie des deux sexes : dix mois au maximum ( $88 \%$ d'une année). Ces mesures synthétiques de la différence entre sexes ne révélent par ailleurs guère d'écarts significatifs entre les expériences de mortalité résumées par les tables de mortalité de 1899-1902 et celles de la table de 1910-1912.

La situation définie par la table de mortalité de 1921-1922 représente par contre une rupture entre le schéma observé au début du siècle et celui des années ultérieures, rupture qui se manifeste dans la différenciation de l'évolution de la dissemblance selon l'âge. Les deux tables antérieures à celle de 1921-1922 et les cinq tables postérieures à cette dernière forment deux groupes à forte corrélation interne : le coefficient de corrélation entre les mesures de dissemblance obtenues de chaque table à partir de celle de 1930-1932 varie entre 0,96 et 0,99 , et pour les deux tables du début du siècle, le coefficient est égal à 0,94 .

La table de 1921-1922 représente ainsi, du point de vue des mesures introduites ici, une phase de transition. On y retrouve en fait pour la dernière fois un accroissement de $1 \mathrm{a}$ dissemblance entre 20 et 40 ans, c'est-à-dire à travers toute la période de vie féconde de la femme, et cela très probablement parce qu'à partir de cette époque, les risques de décès plus élevés chez les hommes à cause de facteurs reliés à l'activité économique ne sont plus contre-balancés, chez les femmes, par les risques de décès imputables à la maternité. Par ailleurs, l'évolution de l'indice aux âges infantiles et juvéniles est plus proche de celle observée à partir des tables ultérieures qu'à partir de celle qui se dégage des tables du début du siècle.

La troisième phase est caractérisée par une croissance continue et considérable de la dissemblance, croissance qui manifeste une première accélération entre les années 1920 et les années 1930, comme en témoignent les indices relatifs à la table de 1930-1932, qui sont environ deux fois plus élevés que ceux obtenus à partir de la table de 1921-1922. La tendance se ralentit par la suite, pour reprendre avec encore plus d'intensité entre les années 1950 et les années 1960, et ralentir à nouveau au cours de la décennie suivante.

Les derniers résultats disponibles, c'est-à-dire ceux relatifs à la table de 1977-1979, mettent en évidence qu'entre les ensembles représentatifs du "nombre d'années qui restent à vivre" aux hommes et aux femmes, il y a désormais une distance (entre les sexes) variant de 6 ans et sept mois à six ans 
lorsqu'on passe de 1 'âge 0 à 1 'âge 25 , et de 5 ans et dix mois à 5 ans et deux mois lorsqu'on passe de 1'âge 30 à l'âge 50 . Etant donné les plus bas niveaux de mortalité pour les femmes, ces distances expriment - comme nous $I^{\prime}$ avons souligné précédemment - de manière synthétique de combien sont supérieures les durées de survie de ce groupe aux divers âges. Avec l'augmentation de la dissemblance s'est modifiée l'évolution en fonction de l'âge, qui, comme le montre la figure 5, s'est uniformisée selon une tendance décroissante selon l'âge, la décroissance étant particulièrement forte après l'âge de 15 ans. L'effet sélectif de la mortalité tend à réduire, d'un âge à l'autre, les distances entre les ensembles représentatifs des durées de survie des deux sexes, mais, avec le passage du temps, les distances, pour un âge donné, tendent à augmenter.

Cette approche suppose une correspondance entre les évolutions de la dissemblance selon l'âge propres à chaque sexe et leur évolution dans le temps, et c'est bien ce qui se vérifie lorsque $1^{\prime}$ on compare les tableaux 2 et 3 . Ces deux tableaux présentent l'évolution temporelle de la dissemblance (en années) entre le "nombre d'années qui restent à vivre" à deux âges successifs, et ceci pour les hommes et les femmes respectivement.

Dans les deux cas, lorsque I'on examine l'évolution selon l'âge, la dissemblance entre les durées de survie de deux groupes quinquennaux d'âge successifs s'est considérablement uniformisée, surtout au cours de la première moitié du siècle : pour les hommes le coefficient de variation entre les indices de dissemblance est passé de $57 \%$ en 1899-1902 à $14 \%$ en 1950-1953, et à moins de $11 \%$ en 1977-1979, les chiffres correspondants pour les femmes étant de $54 \%, 12 \%$ et $6 \%$. Chez les femmes, la dissemblance entre les durées de survie pour deux groupes quinquennaux d'âge successifs était, en 1977-1979, d'un peu moins de cinq ans pour la distance entre les deux premiers groupes, et diminuait progressivement jusqu'à un écart d'un peu plus de quatre ans pour les deux derniers groupes. Chez les hommes, la dissemblance était de 4 ans et 9 mois entre les deux premiers groupes d'âge, et après quelques fluctuations, elle ne commençait à baisser qu'après 20 ans, pour se retrouver avec un écart entre les deux derniers groupes nettement moins élevé que chez les femmes ( 3 ans et 4 mois au lieu de 4 ans et un mois). 
Tableau 2

Dissemblance (en années) entre les variables "nombre d'années qui restent à vivre à compter du $x$-ième anniversaire" relatives à deux groupes d'âge successifs.

Italie, 1899-1902 à 1977-1979. Hommes

\begin{tabular}{|c|c|c|c|c|c|c|c|c|}
\hline$D\left(z_{x}, z_{x+5}\right)$ & $1899-1902$ & $1910-1912$ & $1921-1922$ & $1930-1932$ & $1950-1953$ & $1960-1962$ & $1970-1972$ & $1977-1979$ \\
\hline$D\left(z_{0}, z_{5}\right)$ & 13,14 & 11,33 & 10,37 & 8,87 & 6,02 & 5,31 & 4,93 & 4,78 \\
\hline $\mathrm{D}\left(\mathrm{z}_{5}, \mathrm{z}_{10}\right)$ & 3,67 & 3,93 & 4,12 & 4,48 & 4,74 & 4,89 & 4,84 & 4,89 \\
\hline D $\left(z_{10}, z_{15}\right)$ & 4,23 & 4,33 & 4,35 & 4,48 & 4,76 & 4,83 & 4,84 & 4,88 \\
\hline$D\left(z_{15}, z_{20}\right)$ & 3,96 & 4,00 & 4,03 & 4,23 & 4,67 & 4,69 & 4,72 & 4,75 \\
\hline D $\left(z_{20}, z_{25}\right)$ & 3,69 & 3,79 & 3,85 & 4,06 & 4,60 & 4,67 & 4,71 & 4,81 \\
\hline$D\left(z_{25}, z_{30}\right)$ & 3,74 & 3,79 & 3,89 & 4,11 & 4,59 & 4,68 & 4,74 & 4,78 \\
\hline$D\left(z_{30}, z_{35}\right)$ & 3,86 & 3,90 & 3,98 & 4,11 & 4,58 & 4,66 & 4,73 & 4,77 \\
\hline$D\left(z_{35}, z_{40}\right)$ & 3,88 & 3,92 & 4,02 & 4,08 & 4,54 & 4,60 & 4,64 & 4,70 \\
\hline$D\left(z_{40}, z_{45}\right)$ & 3,79 & 3,87 & 3,99 & 4,02 & 4,38 & 4,48 & 4,49 & 4,56 \\
\hline$D\left(z_{45}, z_{50}\right)$ & 3,73 & 3,78 & 3,88 & 3,92 & 4,15 & 4,29 & 4,28 & 4,32 \\
\hline$D\left(z_{50}, z_{55}\right)$ & 3,54 & 3,67 & 3,76 & 3,75 & 3,89 & 4,01 & 4,02 & 4,00 \\
\hline$D\left(z_{55}, z_{60}\right)$ & 3,41 & 3,46 & 3,51 & 3,54 & 3,62 & 3,63 & 3,73 & 3,73 \\
\hline$D\left(z_{60}, z_{65}\right)$ & 3,01 & 3,17 & 3,24 & 3,24 & 3,37 & 3,26 & 3,36 & 3,33 \\
\hline $\begin{array}{l}\text { Coefficient de } \\
\text { variation }(\%)\end{array}$ & 57,01 & 46,20 & 39,88 & 30,58 & 13,96 & 11,81 & 10,38 & 10,66 \\
\hline
\end{tabular}


Tableau 3

Dissemblance (en années) entre les variables "nombre d'années qui restent à vivre à compter du $x$-ième anniversaire" relatives à deux groupes d'âge successifs.

$$
\text { Italie, 1899-1902 à 1977-1979. Femmes }
$$

\begin{tabular}{|c|c|c|c|c|c|c|c|c|}
\hline$D\left(z_{x}, z_{x+5}\right)$ & $1899-1902$ & $1910-1912$ & $1921-1922$ & $1930-1932$ & $1950-1953$ & $1960-1962$ & $1970-1972$ & $1977-1979$ \\
\hline$D\left(z_{0}, z_{5}\right)$ & 12,54 & 10,94 & 9,88 & 8,77 & 6,32 & 5,63 & 5,34 & 4,97 \\
\hline $\mathrm{D}\left(\mathrm{z}_{5}, \mathrm{z}_{10}\right)$ & 3,54 & 3,77 & 4,11 & 4,55 & 4,87 & 5,08 & 5,23 & 4,92 \\
\hline$D\left(z_{10}, z_{15}\right)$ & 4,08 & 4,20 & 4,28 & 4,48 & 4,82 & 4,87 & 4,91 & 4,92 \\
\hline$D\left(z_{15}, z_{20}\right)$ & 3,79 & 3,85 & 3,94 & 4,18 & 4,75 & 4,86 & 4,88 & 4,90 \\
\hline$D\left(z_{20}, z_{25}\right)$ & 3,56 & 3,66 & 3,78 & 4,02 & 4,69 & 4,84 & 4,86 & 4,89 \\
\hline$D\left(z_{25}, z_{30}\right)$ & 3,56 & 3,68 & 3,81 & 4,06 & 4,66 & 4,80 & 4,85 & 4,89 \\
\hline$D\left(z_{30}, z_{35}\right)$ & 3,64 & 3,74 & 3,92 & 4,14 & 4,64 & 4,76 & 4,82 & 4,86 \\
\hline$D\left(z_{35}, z_{40}\right)$ & 3,69 & 3,80 & 3,94 & 4,13 & 4,59 & 4,70 & 4,76 & 4,81 \\
\hline$D\left(z_{40}, z_{45}\right)$ & 3,77 & 3,90 & 4,01 & 4,14 & 4,53 & 4,63 & 4,69 & 4,74 \\
\hline$D\left(z_{45}, z_{50}\right)$ & 3,90 & 3,95 & 4,02 & 4,11 & 4,40 & 4,52 & 4,55 & 4,62 \\
\hline$D\left(z_{50}, z_{55}\right)$ & 3,77 & 3,85 & 3,89 & 3,98 & 4,26 & 4,38 & 4,60 & 4,47 \\
\hline$D\left(z_{55}, z_{60}\right)$ & 3,64 & 3,70 & 3,75 & 3,78 & 4,06 & 4,17 & 4,05 & 4,31 \\
\hline$D\left(z_{60}, z_{65}\right)$ & 3,15 & 3,32 & 3,40 & 3,47 & 3,78 & 3,93 & 4,01 & 4,07 \\
\hline $\begin{array}{l}\text { Coefficient de } \\
\text { variation }(\%)\end{array}$ & 54,45 & 44,20 & 36,77 & 28,66 & 12,26 & 8,56 & 7,75 & 5,65 \\
\hline
\end{tabular}


Cette uniformisation est surtout due à la baisse considérable de la dissemblance entre les durées de survie des deux premiers groupes d'âge. La chute de la mortalité infantile a été telle qu'on est passé d'un écart d'environ 13 ans entre les durées de survie des deux premiers groupes d'âge, en 1899-1902, à un écart de moins de 5 ans en 1977-1979, ce qui rejoint l'écart observé entre les autres groupes d'âge, écart qui, pour ces derniers, a eu tendance à augmenter, dans une moindre mesure cependant qu'en ce qui concerne les deux premiers groupes d'âge. On peut donc conclure qu'à partir de la fin des années 1970, l'âge "zéro" ne semble plus conditionner dans une mesure aussi considérable que dans le passé la distribution du "nombre d'années qui restent à vivre à compter du x-ième anniversaire" relative à chaque sexe.

\section{QUELQUES CARACTERISTIQUES DE LA MORTALITE DIFFÉRENTIELLE PAR SEXE}

La différenciation et la divergence progressives des niveaux italiens de mortalité des deux sexes qui, à partir des années 1950 , se sont traduites par une surmortalité masculine à tous les âges et par une forte augmentation de cette surmortalité, se sont manifestées dans un contexte d'amélioration continuelle des conditions de mortalité et d'uniformisation généralisée des conditions de vie des femmes et des hommes. Ces caractéristiques de la mortalité différentielle, qui valent pour tous les pays industrialisés, renforcent 1 'hypothèse déjà ancienne (voir Federici, 1950 et 1954) selon laquelle une telle évolution serait le reflet d'une plus grande capacité d'adaptation de la part des femmes aux variations des conditions environnementales au sens large, d'autres facteurs pouvant, selon les circonstances, renforcer l'effet de ce facteur fondamental.

Notre approche en termes de dissemblance semble particulièrement appropriée du point de vue conceptuel pour appréhender cette différence dans le niveau d'adaptation des deux sexes, et, du point de vue' méthodologique, suffisamment souple pour élaborer des schémas de référence permettant de préciser ces deux régimes différents de mortalité.

Commençons par dégager les caractéristiques de longue période. Si notre période d'analyse est articulée selon des sous-périodes de vingt ans, ce qui demeure compatible avec les tables de mortalité disponibles, alors la divergence dans 
l'évolution de la mortalité des deux sexes devient encore plus évidente.

Dans le contexte du déclin de la mortalité que $I^{\prime}$ on a connu au cours de ce siècle, on peut distinguer deux phases, reliées aux causes de cette chute de la mortalité. La première, qui dans le cas de l'Italie s'est prolongée jusqu'au milieu du siècle, reflète $l^{\prime}$ efficacité dont ont fait preuve, dans la lutte contre la mort, les mesures et interventions en matière d'hygiène publique et de prophylaxie. La seconde, qui commence au début des années 1950, est l'expression d'un contrôle plus efficace de la mortalité par maladie infectueuse et par maladie de l'appareil respiratoire, ceci en relation avec le développement de la chemiothérapie. Notre découpage en trois périodes de vingt ans permet de couvrir les étapes terminales de la première phase (1910-1912 à 1930-1932 et 1930-1932 à 1950-1953) et d'esquisser les premiers développements de la seconde (1950-1953 à 1970-1972).

En ce qui concerne la première phase, les hommes et les femmes présentent des évolutions similaires, même si les résultats témoignent toujours d'un niveau (en termes de durée de survie) plus favorable à ces dernières. Comme le montre le tableau 4, la dissemblance (qui représente donc dans tous les cas le gain en durée de survie réalisé au cours de chaque souspériode de vingt ans) décrổt avec l'âge, et, pour un âge donné, elle augmente d'une sous-période à l'autre, du moins jusqu'à la quarantaine; après cet âge, la dissemblance décrô̂t entre les deux sous-périodes.

Au cours de la seconde phase, si l'évolution masculine reste conforme à celle de la phase antérieure (mais avec des gains en durée de survie plus faibles), par contre, dans le cas des femmes, non seulement $n^{\prime}$ observe-t-on plus la forte décroissance de la dissemblance avec l'âge, mais en outre peut-on déceler un renversement de la tendance antérieure selon laquelle les augmentations dans les gains de durée de survie étaient relativement plus fortes aux âges infantiles et juvéniles.

Pour obtenir une vision d'ensemble de la croissance différentielle de la durée de survie pour les deux sexes, on peut se référer à 1 a figure 6 , qui illustre ce que représente en pourcentage le "gain" (en termes de durée de survie) réalisé par les hommes par rapport à celui réalisé par les femmes, et ceci par âge et pour chacune des trois sous-périodes considérées. 
Tableau 4

Dissemblance (en années) entre les variables "nombre d'années qui restent à vivre à compter du $x$-tème anniversairell au cours de trois sous-périodes de vingt ans.

Italie, 1910-1912 à 1970-1972

\begin{tabular}{|c|c|c|c|c|c|c|c|c|c|}
\hline \multirow[b]{2}{*}{ Age } & \multicolumn{3}{|c|}{$1910-1912$ à $1930-1932$} & \multicolumn{3}{|c|}{$1930-1932$ à $1950-1953$} & \multicolumn{3}{|c|}{$1950-1953$ à $1970-1972$} \\
\hline & $\mathrm{F}$ & $\mathrm{H}$ & $\begin{array}{l}\mathrm{H} / \mathrm{F} \\
(\%)\end{array}$ & $\mathrm{F}$ & $\mathrm{H}$ & $\begin{array}{l}\mathrm{H} / \mathrm{F} \\
(\%)\end{array}$ & $\mathrm{F}$ & $\mathrm{H}$ & $\begin{array}{l}\mathrm{H} / \mathrm{F} \\
(\%)\end{array}$ \\
\hline 0 & 8,67 & 7,19 & 82,9 & 11,24 & 9,95 & 88,5 & 7,64 & 5,26 & 68,8 \\
\hline 1 & 7,68 & 6,10 & 79,4 & 9,09 & 7,58 & 83,4 & 5,35 & 2,85 & 53,3 \\
\hline 5 & 5,09 & 3,51 & 68,9 & 6,23 & 4,78 & 76,7 & 4,41 & 1,94 & 44,0 \\
\hline 10 & 4,46 & 2,98 & 66,8 & 5,72 & 4,30 & 75,2 & 4,26 & 1,80 & 42,2 \\
\hline 15 & 4,18 & 2,83 & 67,7 & 5,38 & 4,02 & 74,7 & 4,17 & 1,72 & 41,2 \\
\hline 20 & 3,85 & 2,60 & 67,5 & 4,81 & 3,58 & 74,4 & 4,04 & 1,67 & 41,3 \\
\hline 25 & 3,49 & 2,20 & 63,0 & 4,14 & 3,04 & 73,4 & 3,87 & 1,56 & 40,3 \\
\hline 30 & 3,11 & 1,88 & 60,4 & 3,54 & 2,56 & 72,3 & 3,68 & 1,41 & 38,3 \\
\hline 35 & 2,71 & 1,67 & 61,6 & 3,04 & 2,09 & 68,7 & 3,50 & 1,26 & 36,0 \\
\hline 40 & 2,38 & 1,51 & 63,4 & 2,58 & 1,63 & 63,2 & 3,33 & 1,16 & 34,8 \\
\hline 45 & 2,14 & 1,36 & 63,5 & 2,19 & 1,27 & 58,0 & 3,17 & 1,05 & 33,1 \\
\hline 50 & 1,98 & 1,22 & 61,6 & 1,90 & 1,04 & 54,7 & 3,02 & 0,92 & 30,5 \\
\hline 55 & 1,85 & 1,14 & 61,6 & 1,62 & 0,90 & 55,5 & 2,88 & 0,79 & 27,4 \\
\hline 60 & 1,77 & 1,06 & 59,9 & 1,34 & 0,82 & 61,2 & 2,69 & 0,68 & 25,3 \\
\hline 65 & 1,62 & 0,99 & 61,1 & 1,03 & 0,69 & 67,0 & 2,46 & 0,69 & 28,6 \\
\hline
\end{tabular}

Note : Les chiffres expriment (en années) le "gain» en durée de survie réalisé entre le début et la fin de chaque sous-période de vingt ans. 
Figure 6

Augmentation (entre deux tables de mortalité) de la durée de survie des hommes, en pourcentage de l'augmentation observée pour les femmes, par âge.

Italie, 1910-1912 à 1970-1972

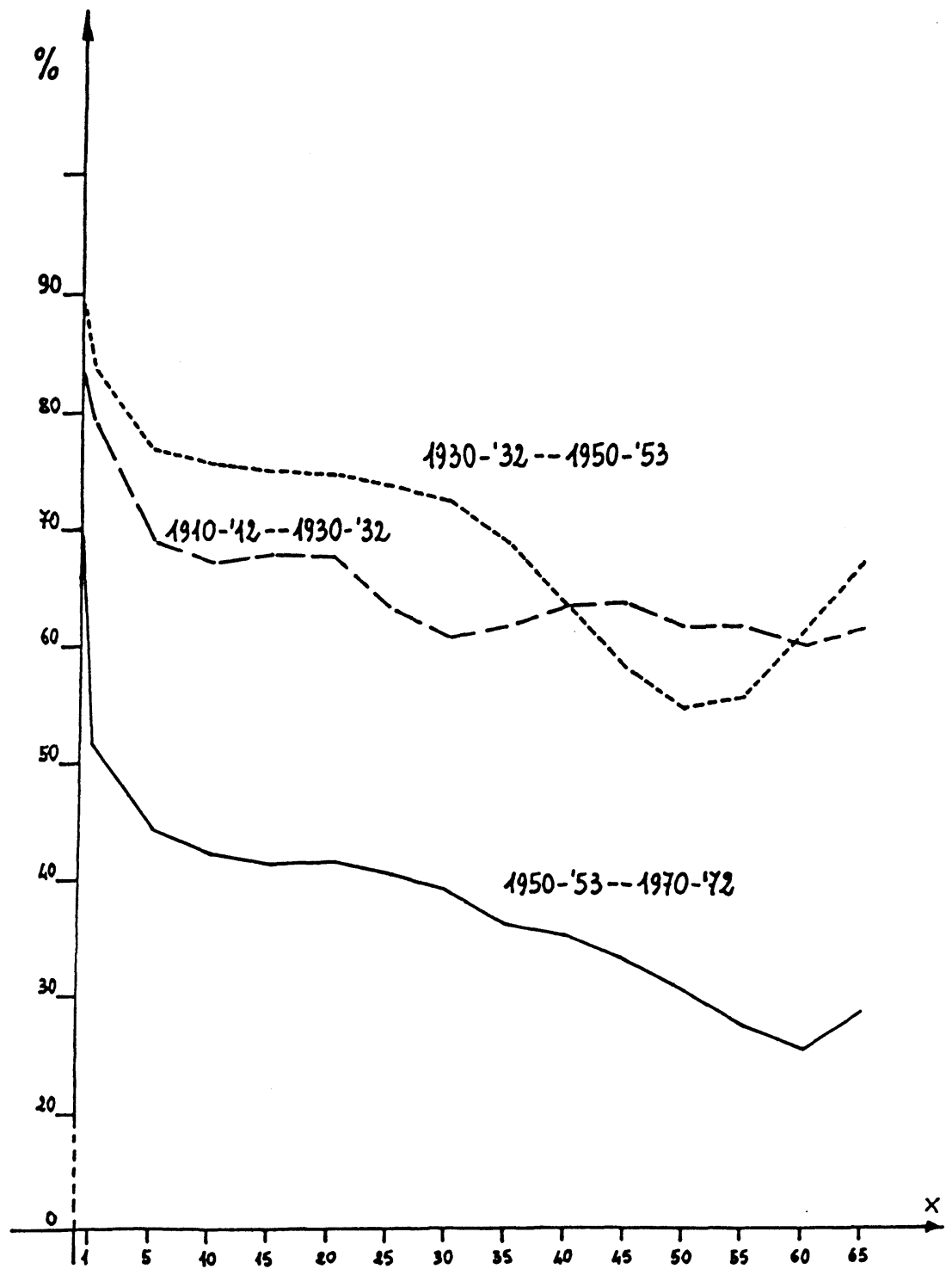


De l'examen de la figure 6 et de la comparaison entre les tableaux 1 et 4 , on peut dégager deux résultats intéressants :

$1^{\circ}$ au cours de la première phase - celle des interventions en matière d'hygiène et de prophylaxie - c'est à l'intérieur de chaque sexe, à chaque âge, que ce sont manifestées les dissemblances (les "gains" en durée de survie) les plus fortes, beaucoup plus qu'entre sexes;

$2^{\circ}$ au cours de la seconde phase - qui, entre autres, se caractérise par une plus grande similitude entre les comportements masculin et féminin - on observe une divergence beaucoup plus forte entre les niveaux de mortalité des deux sexes, même $s^{\prime} i l$ y a encore des gains (relativement faibles par rapport aux périodes antérieures) en termes de durée de survie à chaque âge pour chaque sexe.

Si nous acceptons 1'hypothèse selon laquelle, à la base de la mortalité différentielle selon le sexe, il y a une moindre résistance organique des hommes à l'égard des variations environnementales au sens large, alors, dans un contexte d'uniformisation progressive des conditions de vie des hommes et des femmes, les manifestations de la mortalité différentielle devraient pouvoir se réduire aux seules différences "intrinsèques" de mortalité. C'est à cela que nous consacrerons les dernières pages de cette brève analyse de l'évolution temporelle de la mortalité différentielle selon le sexe.

La mesure de la dissemblance $\Delta$ peut, d'une manière générale, être "normalisée" en la rapportant à sa propre valeur maximale $\left[\max \mathrm{D}\left(\mathrm{Z}_{\mathrm{X}}^{(\mathrm{H})}, \mathrm{Z}_{\mathrm{X}}^{(\mathrm{F})}\right)\right]$, ce qui donne

$$
\Delta^{*}=\frac{\mathrm{D}\left(\mathrm{Z}_{\mathrm{x}}^{(\mathrm{H})}, \mathrm{Z}_{\mathrm{x}}^{(\mathrm{F})}\right)}{\max \mathrm{D}\left(\mathrm{Z}_{\mathrm{x}}^{(\mathrm{H})}, \mathrm{Z}_{\mathrm{x}}^{(\mathrm{F})}\right)}
$$

avec $0 \leqq \Delta^{*} \leqq 1$.

Le problème à résoudre est alors celui de la configuration de cette distance extrême entre $\mathrm{z}_{\mathrm{x}}^{(\mathrm{H})}$ et $\mathrm{Z}_{\mathrm{x}}^{(\mathrm{F})}$, qui doit être celle de la distance maximale "réelle", et non un schéma de référence purement formel qui, entre autres, serait destiné à rester - indûment - constant dans le temps. 
Une solution à ce problème peut être obtenue à partir d'un modèle de mortalité qui, outre sa capacité d'ajustement aux données de la période considérée, permettrait de "déformer" les situations observées aux positions extrêmes. Parmi les modèles théoriques disponibles, celui de Petrioli et Berti (1979) nous a semblé répondre le mieux à notre objectif, car il considère un très large éventail des conditions de mortalité, soit supposées, soit ancrées dans des paramètres déterminés sur des bases empiriques. La situation de $\max \mathrm{D}\left(\mathrm{Z}_{\mathrm{x}}^{(\mathrm{H})}, \mathrm{Z}_{\mathrm{x}}^{(\mathrm{F})}\right)$ a alors été établie en construisant pour chaque table considérée deux modèles de survie, déterminés à partir des valeurs historiquement observées pour les trois indices caractéristiques retenus, à savoir le taux de mortalité infantile, la valeur maximale de la résistance qu'a manifestée la collectivité de la table à l'égard de sa propre disparition, et l'âge auquel a été observée cette valeur maximale. De ces deux modèles de survie, celui relatif aux hommes traduit la situation observée en régime de mortalité élevée, celui relatif aux femmes réfère à la situation observée en régime de mortalité faible.

Les résultats du calcul de $1^{\prime}$ indice normalisé $\Delta^{*}$ sont présentés dans le tableau 5 et la figure 7 . On y observe, comme précédemment, une surmortalité masculine à tous les âges à partir des années 1950-1953, et pour les années antérieures, dès le groupe d'âge de 35 ans et plus. Ce n'est toutefois qu'à partir des années 1970 que les écarts entre sexes demeurent relativement stables à travers les âges. De ce point de vue, il nous semble que $I^{\prime}$ on puisse affirmer que le processus de différenciation de la mortalité selon les sexes se soit manifesté après les années 1920, mais à cette époque, et ce jusqu'aux années 1960, cette différenciation reflétait dans le cas des hommes une forte composante de mortalité pour cause professionnelle, comme le laisse percevoir la croissance plus rapide de l'indice au cours des âges terminaux de la vie active.

\section{CONCLUSION}

Les résultats que nous avons obtenus en exprimant la mortalité différentielle en termes de dissemblance entre les variables "nombre d'années qui restent à vivre à compter du $x$-ième anniversaire" des deux sexes, nous conduisent à avancer que la période la plus récente est aussi la plus uniformément "dichotomisée", en termes relatifs, en ce qui concerne les conditions de mortalité des deux sexes. Etant donné la richesse 
Indice normalisé de dissemblance entre les variables "nombre d'années qui restent à vivre à compter du x-ième anniversaire" relatives aux deux sexes, selon l'âge. Italie, 1899-1902 à 1977-1979

\begin{tabular}{rcccccccc}
\hline Age & $1899-1902$ & $1910-1912$ & $1921-1922$ & $1930-1932$ & $1950-1953$ & $1960-1962$ & $1970-1972$ & $1977-1979$ \\
\hline 0 & 0,038 & 0,052 & 0,093 & 0,136 & 0,319 & 0,497 & 0,687 & 0,827 \\
1 & 0,047 & 0,044 & 0,044 & 0,093 & 0,276 & 0,482 & 0,718 & 0,904 \\
5 & 0,054 & 0,058 & 0,052 & 0,129 & 0,322 & 0,526 & 0,746 & 0,918 \\
10 & 0,061 & 0,075 & 0,061 & 0,146 & 0,355 & 0,550 & 0,769 & 0,928 \\
15 & 0,067 & 0,070 & 0,071 & 0,166 & 0,383 & 0,569 & 0,786 & 0,936 \\
20 & 0,072 & 0,077 & 0,086 & 0,186 & 0,409 & 0,568 & 0,785 & 0,927 \\
25 & 0,088 & 0,089 & 0,090 & 0,213 & 0,436 & 0,566 & 0,784 & 0,916 \\
30 & 0,101 & 0,105 & 0,104 & 0,243 & 0,467 & 0,573 & 0,788 & 0,914 \\
35 & 0,137 & 0,131 & 0,127 & 0,250 & 0,505 & 0,585 & 0,796 & 0.912 \\
40 & 0,200 & 0,166 & 0,149 & 0,278 & 0,552 & 0,602 & 0,801 & 0,919 \\
45 & 0,253 & 0,189 & 0,179 & 0,302 & 0,587 & 0,619 & 0,797 & 0,915 \\
50 & 0,268 & 0,189 & 0,180 & 0,320 & 0,610 & 0,634 & 0,783 & 0,898 \\
55 & 0,225 & 0,185 & 0,184 & 0,336 & 0,609 & 0,634 & 0,754 & 0,858 \\
60 & 0,145 & 0,137 & 0,159 & 0,373 & 0,587 & 0,611 & 0,703 & 0,807 \\
65 & 0,120 & 0,092 & 0,136 & 0,435 & 0,577 & 0,551 & 0,626 & 0,731 \\
\hline
\end{tabular}


Figure 7

Indice normalisé de dissemblance entre les variables "nombre d'années qui restent à vivre à compter du $\mathrm{x}$-ième anniversaire" relatives aux deux sexes, selon 1'âge. Italie, 1899-1902 à 1977-1979

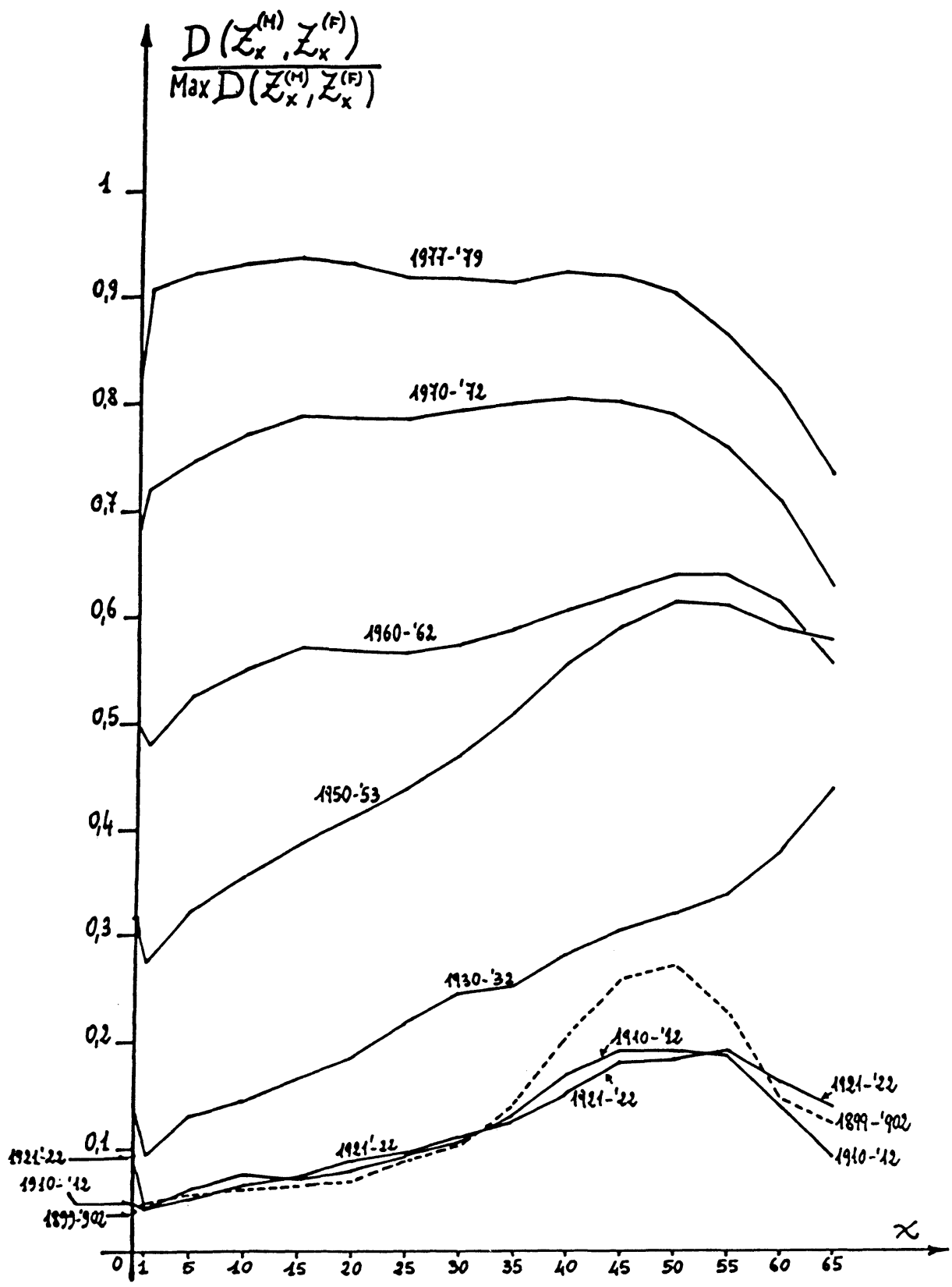


de l'information statistique disponible sur les causes de décès de cette période plus récente, on peut donc conclure que le moment est venu d'approfondir, sur la base des données les plus récentes, l'examen du rôle des facteurs biologiques, physiologiques et sociaux dans la surmortalité masculine.

\section{REFFERENCES BIBLIOGRAPHIQUES}

CIFARELLI, D.M. et F. MOELLER, 1981. Lezioni di Statistica descrittiva. Milano, Cisalpino-Goliardica.

DI COMITE, Luigi, 1974. La mortalità in Italia. Roma, Istituto di Demografia.

FEDERICI, Nora, 1950. "La mortalità differenziale dei due sessi e le sue possibile causel. Statistica, 3 .

FEDERICI, Nora, 1954. ROsservazioni sul1' evoluzione di alcune caratteristiche della mortalità e sul problema della supermortalità maschilel. Bulletin de l'Institut international de statistique, 34,3 .

GINI, Corrado, 1914. "Di una misura della dissomiglianza e delle sue applicazioni allo studio delle relazioni statistichel. Atti dell' Istituto Veneto di Arti, Scienze, Lettere, 74 .

LETI, G., 1983. Statistica descrittiva. Bologna, I1 Mulino.

MOOD, A.M., F.A. GRAYBILL and D.G. BOES, 1974. Introduction to the Theory of Statistics. New York-Tokyo, McGraw HillKogakusha.

NATALE, Marcello, 1979. Caratteristiche e tendenze della supermortalità maschile. Roma, Istituto di Demografia.

PETRIOLI, L. et M. BERTI, 1979. Model11 di mortalità. Milano, F. Angeli. 


$$
\text { RESUME - SUMMARY - RESUMEN }
$$

MACCHERONI Carlo - LA MORTALITÉ DIFFÉRENTIELLE MESURÉE EN TERMES DE DISSEMBLANCE. LES ECARTS DE MORTALITE ENTRE SEXES SELON LES TABLES ITALIENNES DU XXe SIECLE

La comparaison des espérances de vie peut, sous certaines conditions, conduire à une mesure bien plus précise, à savoir celle de la dissemblance entre les distributions empiriques des durées de survie mises en confrontation. Cet article examine les conditions qui doivent être réunies pour qu'on puisse accepter cette interprétation de la comparaison des espérances de vie, et applique cette approche aux données des tables de mortalité italiennes du XXe siècle.

MACCHERONI Car1O - DIFFERENTIALS IN MORTALITY MEASURED IN TERMS OF DISSIMILARITY. MORTALITY DIFFERENCES BETWEEN SEXES ACCORDING TO THE ITALIAN TABLES OF THE 20th CENTURY

Under some conditions, the comparison of life expectancies may lead to a much more accurate measure, in terms of dissimilarity between the empirical distributions of the number of years expected to live at each age. This paper analyses these conditions and applies the dissimilarity approach to the data of the Italian life tables of the 20 th century.

MACCHERONI Car1o - LA MORTAIIDAD DIFERENCIAL MEDIDA EN TÉRMINOS DE DESEMEJANZA. LAS DIFERENCIAS DE MORTALIDAD ENTRE LOS SEXOS SEGÚN LAS TABLAS ITALIANAS DEL SIGLO XX

La comparación de las esperanzas de vida puede, bajo algunas condiciones, conducir a una medida mucho más precisa, o sea la desemejanza entre las distribuciones empiricas de las duraciones de supervivencia puestas en confrontación. Este artículo examina las condiciones que deben reunirse para poder aceptar esta interpretación de la comparación de las esperanzas de vida y aplica este enfoque a los datos de las tablas italianas del siglo $\mathrm{XX}$. 